\title{
Degradation of Chitin and Chitosan by a Recombinant Chitinase Derived from a Virulent Aeromonas hydrophila Isolated from Diseased Channel Catfish
}

\author{
Dunhua Zhang1* ${ }^{*}$ John M. Bland ${ }^{2}$, Dehai Xu' ${ }^{1}$, Siyin Chung ${ }^{2}$ \\ ${ }^{1}$ Aquatic Animal Health Research Unit, USDA-ARS, Auburn, USA \\ ${ }^{2}$ Southern Regional Research Center, USDA-ARS, New Orleans, USA \\ Email: *dunhua.zhang@ars.usda.gov
}

Received 2 July 2015; accepted 23 August 2015; published 26 August 2015

Copyright (C) 2015 by authors and Scientific Research Publishing Inc.

This work is licensed under the Creative Commons Attribution International License (CC BY). http://creativecommons.org/licenses/by/4.0/

\section{(c) (i) Open Access}

\begin{abstract}
A chitinase was identified in extracellular products of a virulent Aeromonas hydrophila isolated from diseased channel catfish (Ictalurus punctatus). Recombinant chitinase (rChi-Ah) was produced in Escherichia coli. Purified rChi-Ah had optimal activity at temperature of $42^{\circ} \mathrm{C}$ and $\mathrm{pH} 6.5$. The affinity $(\mathrm{Km})$ for chitosan was $4.18 \mathrm{mg} \cdot \mathrm{ml}^{-1}$ with $V_{m a x}$ of $202.5 \mathrm{mg} \cdot \mathrm{min}^{-1} \cdot \mathrm{mg}^{-1}$. With colloidal chitin as substrate, rChi-Ah generated $\mathrm{N}, \mathrm{N}^{\prime}$-diacetyl-glucosamine predominantly. Conversion of chitosan ( $\geq 75 \%$ deacetylated) by rChi-Ah revealed five major products: 2 to 4 units of glucosamine, all of which had at least one acetyl group. It was determined that $\mathrm{N}$-acetylated glucosamine was the recognition and cleavage site of $\mathrm{rChi}-\mathrm{Ah}$; the minimal and maximal cleavages were two and four glucosamine units, respectively. Functional analysis of rChi-Ah suggests that $A$. hydrophilachitinase is a bioactive chitinolytic enzyme, which may benefit the pathogen for survival and/or infection.
\end{abstract}

\section{Keywords}

Aeromonas hydrophila, Recombinant Chitinase, Chitin Degradation, Chitosan Degradation

\section{Introduction}

Chitin is a linear polymer composed of $\beta$-(1 $\rightarrow 4)-\mathrm{N}$-acetyl-D-glucosamine and is predominantly found in ex-

*Corresponding author.

How to cite this paper: Zhang, D., Bland, J.M., Xu, D.H. and Chung, S.Y. (2015) Degradation of Chitin and Chitosan by a Recombinant Chitinase Derived from a Virulent Aeromonas hydrophila Isolated from Diseased Channel Catfish. Advances in Microbiology, 5, 611-619. http://dx.doi.org/10.4236/aim.2015.59064 
oskeletons of crustaceans and insects and cell walls of fungi and yeasts [1]. Chitinases play an important physiological and ecological role as recyclers of chitin, the second most abundant polymers in nature, after cellulose [2]. Microorganisms, particularly bacteria, are major sources of chitinases; chitooligomers produced by bacterial chitinases have gained worldwide research interest for their diverse properties and potential industrial uses [3].

In genus Aeromonas, several species of bacteria were known to produce extracellular chitinases [4]. Chitinases of two environmental strains of A. hydrophila had been partially characterized [5] [6]. Recently, a chitinase was identified in extracellular products (ECP) of a virulent $A$. hydrophila strain, ML-10-51K, and the chitinase was recognized by antibodies in catfish anti-ECP serum [7]. Virulent A. hydrophila has emerged as a major concern in catfish aquaculture in the Southeastern United States since 2009-2010 outbreaks of motile Aeromonas septicemia (MAS; [8]); and ECP of A. hydrophila was found to be associated with its virulence [9]. As part of efforts to understand functions of ECP proteins in A. hydrophila ML-10-51K, this study was to produce recombinant chitinase and evaluate its biochemical properties. The gene encoding the chitinase was cloned and expressed in Escherichia coli. Active recombinant chitinase was purified. Bioconversion of chitin and chitosan (partially de-acetylated chitin) by the enzyme was subsequently determined and some biochemical characteristics of the enzyme were analyzed.

\section{Materials and Methods}

\subsection{Chitinase Gene Cloning and Expression in E. coli}

Genomic DNA of A. hydrophila strain ML-10-51K was isolated using AquPure genomic isolation kit (BioRAD, Hercules, CA, USA). Two primers, targeting the encoding region of mature chitinase peptide (based on prediction of SignalP 4.1 at www.cbs.dtu.dk/services/SignalP), were synthesized according to the sequence of GenBank\# AHML_05229 [10]. The forward primer, ${ }^{5}$ GCAGACCATG GCC GCT CCC GGC AAA CCC ACC A, was flanked with an Nco I restriction site at the 5' end (underlined) while the reversed one, 5'CCTGGCTCGAG TTT GCA GCT GGC CGC GCC GAT GTC, had an Xho I restriction site at 5' end (underlined). The chitinase gene was PCR-amplified using the genomic DNA as template and Advantage 2 Polymerase Mix kit (ClonTech, Mountain View, CA, USA). Following digestion with Nco I and Xho I, the amplicon was cloned into pET28a vector plasmids (Novagen, Madison, WI, USA) at corresponding restriction sites. Recombinant pET28a plasmids were propagated in E. coli NovaBlue cells (Novagen) and purified for sequencing analysis. The cloned chitinase gene did not contain a stop codon at the 3' end so that six consecutive histidine residues (His-tag), embedded in the vector, would fuse to the C-terminus of chitinase during expression.

Competent cells of E. coli Rosetta 2(DE3) (Novagen) were transformed with recombinant pET28a plasmids and selected on LB agar (Fisher Scientific, Fair Lawn, NJ, USA) supplemented with kanamycin $\left(50 \mu \mathrm{g} \cdot \mathrm{ml}^{-1}\right.$ ) and chloramphenicol $\left(34 \mu \mathrm{g} \cdot \mathrm{ml}^{-1}\right)$. Positive colonies were propagated in LB broth with the antibiotics by shaking at 200 revolutions per minute (rpm) at $37^{\circ} \mathrm{C}$. Over-expression of the target gene was induced by addition of Isopropyl $\beta$-D-1-thiogalactopyranoside (IPTG) to the bacterial culture at final concentration of $0.4 \mathrm{mM}$ when optical density $\left(\mathrm{OD}_{600}\right)$ reached to $0.4-0.6$, followed by shake-incubation at $21^{\circ} \mathrm{C}$ for $18-20 \mathrm{~h}$. After induction, bacterial cells were harvested by centrifugation and stored at $-80^{\circ} \mathrm{C}$ until protein purification. Recombinant chitinase produced in E. coli was found present in soluble cytoplastic fraction after cell lysis and was purified under native conditions using ProBond ${ }^{\mathrm{TM}}$ Purification System (Invitrogen, Carlsbad, CA, USA). Buffer-exchange was performed after the protein was eluted from the binding resin using phosphate-buffer saline (PBS; Sigma-Aldrich, St. Louis, MO, USA) and 20 K MWCO-concentrator (Thermo Scientific, Rockford, IL, USA). The final protein concentration was estimated with the Bradford Reagent (Sigma-Aldrich) using bovine serum albumin (BSA) as the standard protein. The recombinant chitinase is referred to rChi-Ah hereafter.

\subsection{Chitosan, Colloidal Chitin and Colloidal Chitin Medium}

Chitosan was purchased from Sigma-Aldrich (deacetylation $\geq 75 \%$ ). Colloidal chitin was prepared from coarse shrimp chitin flakes (Sigma) using modified protocols of Murthy and Bleakley [11] and Seitzman [12]. Briefly, $3 \mathrm{~g}$ of flakes were dissolved in $60 \mathrm{ml}$ of $\mathrm{HCl}(12 \mathrm{~N})$ in chemical fume hood at room temperature with stirring for $2 \mathrm{~h}$, and, then, $240 \mathrm{ml}$ cold $\left(4^{\circ} \mathrm{C}\right)$ ethanol were added while stirring continued, followed by addition of $10 \mathrm{ml}$ of $0.1 \mathrm{M}$ sodium acetate ( $\mathrm{NaAc}$; $\mathrm{pH}$ 6.3). Aliquots of the chitin suspension were dispensed in $50 \mathrm{ml}$ tubes and centrifuged at $5000 \mathrm{rpm}$ for $10 \mathrm{~min}$. Precipitates were washed with 4 volumes of $0.1 \mathrm{M} \mathrm{NaAc}(\mathrm{pH} \mathrm{6.3)}$ ) for 4 times 
by resuspension, votexing and centrifugation. The final colloidal chitin was suspended in $0.1 \mathrm{M}$ NaAc with concentration of about $75 \mathrm{mg} \cdot \mathrm{ml}^{-1}$ (pH 6.3). Colloidal chitin media were prepared by adding colloidal chitin equivalent to $1 \mathrm{~g}$ chitin flakes to $100 \mathrm{ml}$ LB agar suspension (containing $4 \mathrm{~g}$ LB agar in distilled water; referred to CCLB) or $100 \mathrm{ml}$ agar suspension (containing $1.5 \mathrm{~g}$ agar in distilled water; referred to CCA), and poured into $10 \mathrm{~cm}$ Petri dishes after autoclaving.

\subsection{Chitinolytic Activities of A. hydrophila Chitinase and rChi-Ah}

Extracellular chitinase activity of A. hydrophila was assessed by inoculating the bacterial cells onto the CCLB plate. The plate was incubated at $28^{\circ} \mathrm{C}$ until clear (chitinolytic) haloes appeared around the colonies. Similarly, the chitinolytic activity of rChi-Ah was determined by applying $10 \mu \mathrm{l}$ protein solution $\left(1 \mu \mathrm{g} \cdot \mu \mathrm{l}^{-1}\right)$ to a 7 -mm filter paper disk placed on the surface of a CCA plate and the plate was incubated at $37^{\circ} \mathrm{C}$ until halo formation.

\subsection{Thin Layer Chromatography (TLC) and Liquid Chromatography-Mass Spectrometry (LC-MS) Analyses of rChi-Ah Degradation Products}

Colloidal chitin and $0.4 \%$ chitosan dissolved in $100 \mathrm{mM}$ sodium acetate, $\mathrm{pH}$ 6.2, were used as substrates for rChi-Ah degradation. An aliquot of $400 \mu \mathrm{l}$ of colloidal chitin or chitosan in 2-ml centrifuge tube was mixed with $30 \mu \mathrm{l}$ of rChi-Ah $\left(1.2 \mu \mathrm{g} \cdot \mu \mathrm{l}^{-1}\right.$ in PBS). For control, $30 \mu \mathrm{l}$ of PBS were added to the substrate. The mixtures were incubated at $37^{\circ} \mathrm{C}$ with constant shaking at $200 \mathrm{rpm}$ for $24 \mathrm{~h}$. For TLC analysis, $6 \mu \mathrm{l}$ of sample were taken from each mixture and directly spotted on a TLC plate (see below); for LC-MS analysis, the mixtures were individually filtered through a PES membrane (10 kDa MWCO; Pierce-Thermo Scientific) and $10 \mu \mathrm{l}$ of the filtrate were applied to analysis (see below).

TLC conditions were performed with modified methods of Bond et al. [13] as follows: after spotting the samples on the TLC plate (Silica gel 60; EMD Chemicals Inc. Gibbstown, NJ, USA), chromatography was developed in a solvent containing n-butanol, glacial acetic acid and water (at ratio of 2:1:1) for 90 min. The plate, after drying with a stream of warm air, was stained by spraying with $p$-anisaldehyde reagent (ethanol:glacial acetic acid:sulfuric acid: $p$-anisaldehyde $=9.0: 0.1: 0.75: 0.75$ ) and heated in an oven at $115^{\circ} \mathrm{C}$ for about 5 min. Reference standards, N-acetyl-D-glucosamine and N,N'-diacetyl-glucosamine (N,N'-diacetyl-chitobiose), 8 - $10 \mu \mathrm{g}$ each, were included in each TLC run.

LC-MS was carried out on a Waters Alliance (2695) station. The sample was chromatographed using Atlantis Silica HILIC column ( $3 \mu \mathrm{m}, 2.1 \times 50 \mathrm{~mm}$; Waters Corp., Milford, MA, USA) with mobile phases AF $(0.1 \%$ ammonium formate) and ACN (acetonitrile). Each run started at 5\% AF and 95\% ACN with a gradient from 5\% to $50 \% \mathrm{AF}$ over $45 \mathrm{~min}$, followed by a $10 \mathrm{~min}$ isocratic run at $50 \% \mathrm{AF}$. The flow rate was set at $0.3 \mathrm{ml} \cdot \mathrm{min}^{-1}$. Eluting masses ranging from 130 to $2000 \mathrm{~m} / \mathrm{z}$ were detected and analyzed in both positive and negative ESI-MS (electrospray ionization-mass spectrometry) modes.

\subsection{Thermostability, Optima of Temperature and pH, and Kinetics of rChi-Ah}

The heat stability of rChi-Ah was assessed by incubating aliquots of the protein at temperatures ranging from 37 to $62^{\circ} \mathrm{C}$ for $30 \mathrm{~min}$. Aliquots of $4 \mu \mathrm{l}$ treated samples $(4.8 \mu \mathrm{g})$ were then mixed with $50 \mu \mathrm{l} 0.4 \%$ chitosan (described above). The mixtures were incubated at $37^{\circ} \mathrm{C}$ for $1 \mathrm{~h}$. Residue activities of treated rChi-Ah were measured by amounts of reducing oligo-N-acetyl-glucosamines released from chitosan using modified tetrazolium blue assay [14]. Briefly, $10 \mu \mathrm{l}$ of each reaction mixture were dispensed into triplicate wells of a 96-well PCR-plate; $90 \mu \mathrm{l}$ of tetrazolium blue reagents were then mixed into individual sample wells. The plate was placed in PCR thermocycler and incubated with following program: $97^{\circ} \mathrm{C}$ for 4 min and $22^{\circ} \mathrm{C}$ for $5 \mathrm{~min}$. Soon after the thermo-program, $95 \mu \mathrm{l}$ of individual samples were orderly transferred to a new 96-well microplate and the plate was read at absorbance $660 \mathrm{~nm}$ in a microplate reader (xMark, BioRAD, Hercules, CA, USA). Serial dilutions of N,N'-diacetyl-chitobiose, ranging from 50 to $1000 \mu \mathrm{M}$, were included in the assay as reference standards. Data were validated where correlation coefficients $\left(r^{2}\right)$ of the standard curves were equal to or greater than 0.98. Similarly, the effect of different temperatures on rChi-Ah activity was measured by incubating the same composite (protein and chitosan) reaction above, but no pre-heat treatment of the protein, over a range of temperatures from $27^{\circ} \mathrm{C}$ to $62^{\circ} \mathrm{C}$ for $1 \mathrm{~h}$. The effect of $\mathrm{pH}$ on the rChi-Ah activity was determined by incubating the protein with chitosan in different $\mathrm{pH}$ buffers $(4.5$ to 7.5$)$ at $37^{\circ} \mathrm{C}$ for $1 \mathrm{~h}$. All buffers were made up of 100 
$\mathrm{mM} \mathrm{NaAc}$ and $\mathrm{pH}$ values were adjusted with $6 \mathrm{M} \mathrm{NaOH}$ or $5 \mathrm{~N} \mathrm{HCl}$. All assays had three replicates. Oligo-N-acetyl-glucosamines yielded in each reaction were determined using tetrazolium blue assay methods described above.

Kinetics of rChi-Ah was estimated using serially diluted chitosan in $100 \mathrm{mM}$ NaAc (pH 6.3) with 6 concentrations ranging from 0.1 to $5.0 \mathrm{mg} \cdot \mathrm{ml}^{-1}$. Reactions were set up in a 96-well PCR plate. Aliquots of $2 \mu \mathrm{l} \mathrm{rChi-Ah}$ $(2 \mu \mathrm{g})$ were mixed with $23 \mu \mathrm{l}$ of each concentration of chitosan in triplicate wells. The plate was incubated at $37^{\circ} \mathrm{C}$ for $10 \mathrm{~min}$ and at $22^{\circ} \mathrm{C}$ for $5 \mathrm{~min}$ in PCR thermocycler. Aliquots of $10 \mu \mathrm{l}$ of each reaction were orderly transferred to new wells of the same plate and mixed with $90 \mu \mathrm{l}$ of tetrazolium blue reagents, followed by the same procedures described above for thermostability assay. The Michaelis constant $(\mathrm{Km})$ and the maximal reaction rate (Vmax) were determined by the Lineweaver-Burk plot method.

\section{Results}

\subsection{A. hydrophila ML-10-51K Chitinase and Recombinant Chitinase (rChi-Ah)}

The cloned chitinase gene of $A$. hydrophila ML-10-51K was 2562 bp in length, encoding 854 amino acid peptides with calculated molecular weight of $90.55 \mathrm{kDa}$. Amino acid sequence alignment showed that the ML-10-51K chitinase was identical to that of another virulent A. hydrophila strain ML09-119 [10], and shared 99.3\% identity with an American Type Culture Collection (ATCC) strain ATCC7966 [15] and 79.9\% identity with a fresh water strain SUWA [6] (data not shown). All of these chitinases have typical conserved domains, including chitin catalytic domain (glycosyl hydrolase family 18), N-terminal early set domain, and two C-terminal chitin binding domains (data not shown).

Production of recombinant $A$. hydrophila ML-10-51K chitinase in E. coli was achieved using methods described. Apparently homogeneous rChi-Ah was recovered from the crude cell extract following His-tag affinity purification (Figure 1(a)). The electrophoretic mobility of rChi-Ah was similar to the native chitinase identified in extracellular products of $A$. hydrophila ML-10-51K (Figure 1(b)).

\subsection{Chitinolytic Activities of A. hydrophila and rChi-Ah}

Cells of A. hydrophila ML-10-51K were able to secrete chitinase and hydrolyze colloidal chitin, forming clear halo in CCLB medium (left colony of Figure 2(a)). The closely-related strain ATCC7966 also produced similar halo, but the hydrolytic activity appeared to be less in terms of the ratio between halo and colony diameters

(a)

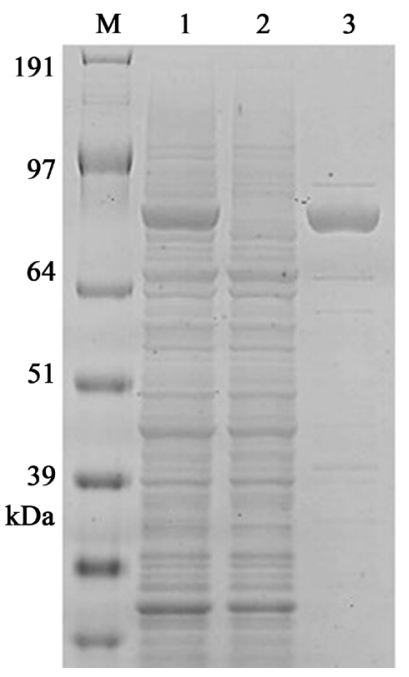

(b)

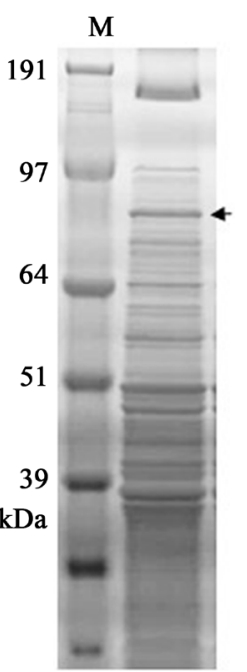

Figure 1. SDS-PAGE of recombinant chitinase (rChi-Ah) and extracellular products (ECP) of A. hydrophila. (a) Production of rChi-Ah; Lane 1: extract of crude cell lysate, Lane 2: ProBond resin unbound proteins, Lane 3: purified rCh-Ah (about 1.5 $\mu$ g loaded). (b) Extracellular product (ECP) of A. hydrophila ML-10-51K; arrow indicates the protein band identified as chitinase by LC-MS/MS analysis (revealed by 41 exclusive unique peptides with $42 \%$ coverage). Lane M: SeeBluePlus 2 Prestained protein standards (Invitrogen). 
(a)

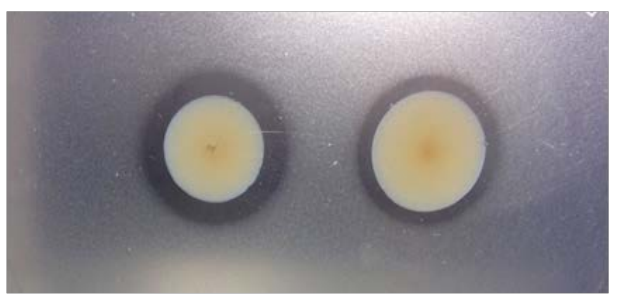

(b)

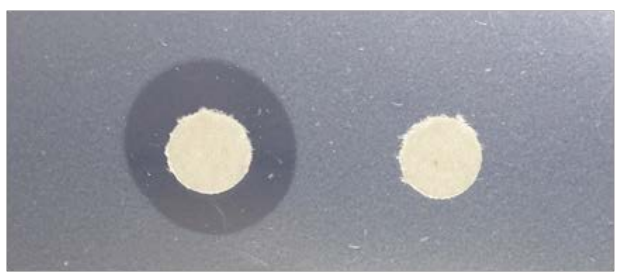

(c)

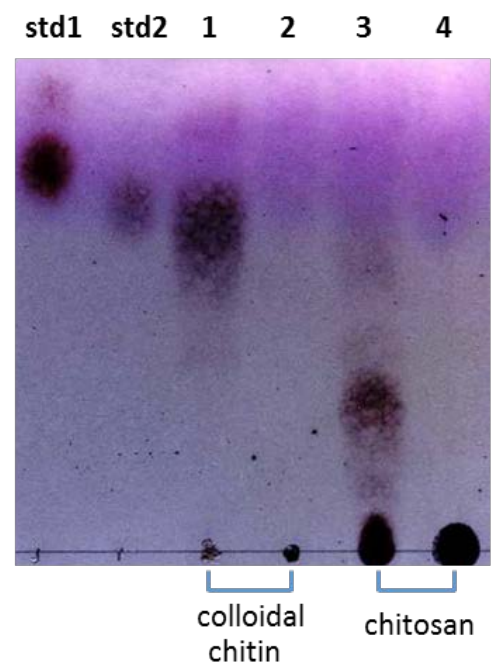

Figure 2. Chitinolytic activities of A. hydrophila chitinase. (a) Clear halos formed around colonies of A. hydrophila ML-10-51K (left) and ATCC7966 (right) in 1\% colloidal chitin LB agar (CCLB). (b) Clear halos formed in 1\% colloidal chitin agar (CCA) by purified rChi-Ah (left; the same sample as in Lane 3 of Figure 1(a)), compared with resin unbound proteins (right; the same sample as in Lane 2 of Figure 1(a)). (c) TLC of colloidal chitin and chitosan degradation products catalyzed by rChi-Ah; std1: N-acetyl-D-glucosamine, std2: N,N'-diacetyl-glucosamine, 1: colloidal chitin with rChi-Ah, 2: colloidal chitin without rChi-Ah, 3, chitosan with rChi-Ah, and 4: chitosan without rChi-Ah.

(right colony of Figure 2(a)).

Purified recombinant chitinase rChi-Ah was enzymatically active and formed clear chitinolytic halo in CCA medium (left paper disk of Figure 2(b)). No activity was observed in resin-unbound proteins of the crude cell extract (right disk of Figure 2(b)).

TLC analysis showed that rChi-Ah could hydrolyze not only colloidal chitin but also partially deacetylated chitosan (Figure 2(c)). One major degradation product was generated from colloidal chitin. The product appeared to be more polar than N-acetyl-glucosamine (reference standard 1 ) and was similar to N,N'-diacetylglucosamine (reference standard 2) in retardation factor (Rf) and color reaction. For chitosan, the rChi-Ah mediated hydrolysis showed one major product and some minors, all of which had lower Rf values than N,N'-diacetyl-glucosamine. Both of controls (colloidal chitin and chitosan without rChi-Ah) had no self-hydrolysis product.

\subsection{Identification of Colloidal Chitin and Chitosan Degradation Products Mediated by rChi-Ah}

LC-MS analysis revealed that the degradation products of colloidal chitin had masses $(\mathrm{m} / \mathrm{z})$ of 425,849 , and 871,

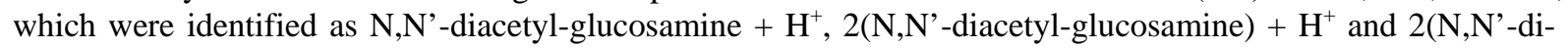
acetyl-glucosamine) $+\mathrm{Na}^{+}$, respectively, while the $\mathrm{m} / \mathrm{z}$ of reference standard $\mathrm{N}, \mathrm{N}^{\prime}$-diacetyl-glucosamine was 871. For chitosan, five degradation products were detected; they had masses of 383, 586, 544, 747, and 705 (in the eluting order), respectively. By molecular calculation, product 1 (P1) is di-glucosamine with one acetyl group, P2 is tri-glucosamine with two acetyl groups, P3 is tri-glucosamine with one acetyl group, P4 is tetraglucosamine with two acetyl groups and P5 is tetra-glucosamine with one acetyl group. Based on the cleavage patterns, the structures of the five products are putatively depicted in Figure 3, showing that N-acetylated glucosamine is the recognition and cleavage site of rChi-Ah; the minimal and maximal cleavages are two and four glucosamine units, respectively. The relative abundance of these five products was in following orders: P3 > P4 > P1 > P2 > P5 and the P3 is likely the major spot seen in TLC (Figure 2(c)).

\subsection{Thermostability, Optima of Temperature and $\mathrm{Ph}$, and Kinetics of $\mathrm{rChi}-\mathrm{Ah}$}

Using chitosan as substrate, the optimal temperature for rChi-Ah activity was around $42^{\circ} \mathrm{C}$. The enzyme was stable 
Glucosamine $\mathrm{N}$-acetyl-Glucosamine

P1:

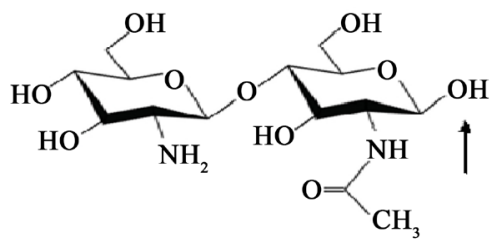

P2:

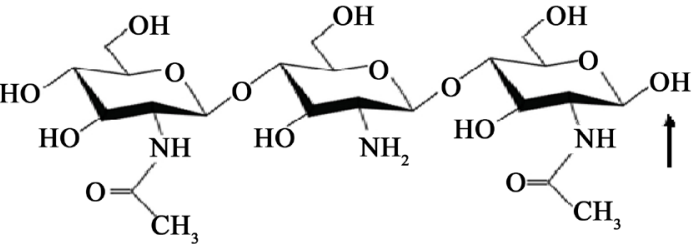

P3:

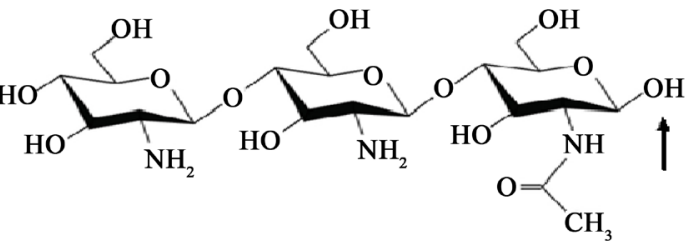

P4:

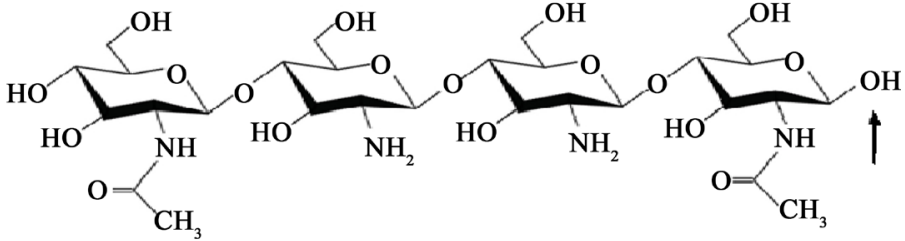

P5

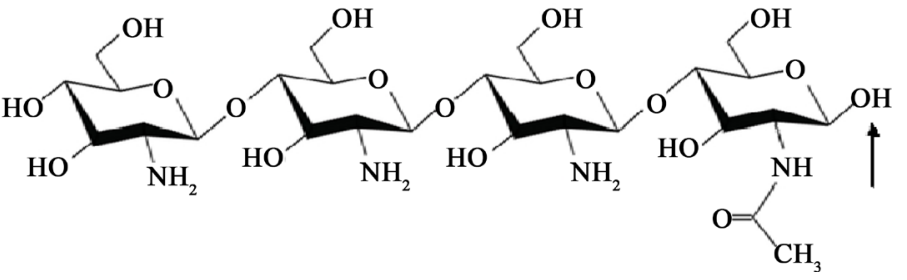

Figure 3. Putative structures of rChi-Ah mediated chitosan degradation products (P1 - P5). Arrows indicate rCh-Ah recognition and cleavage sites ( $\mathrm{N}$-acetylated di- to tetra-glucosamine) and reducing ends which react with tetrazolium reagent.

over temperature $37^{\circ} \mathrm{C}$ to $42^{\circ} \mathrm{C}$; $85 \%$ residual activity remained at $47^{\circ} \mathrm{C}$; and $65 \%$ activity lost at $52^{\circ} \mathrm{C}(\mathrm{Figure}$ 4(a)). The optimal $\mathrm{pH}$ for the enzyme activity was around 6.5 and lower activities were observed with $\mathrm{pH}$ away from the optimal (Figure 4(b)). The catalytic activity of rChi-Ah against chitosan showed a $\mathrm{Km}$ value of 4.18 $\mathrm{mg} \cdot \mathrm{ml}^{-1}$ with Vmax of $202.5(4.05 \times 50) \mathrm{mg} \cdot \mathrm{min}^{-1} \cdot \mathrm{mg}^{-1}$ (Figure 4(c)).

\section{Discussion}

Using recombinant chitinase rChi-Ah, results of this study demonstrated that the virulent A. hydrophila ML-10$51 \mathrm{~K}$ produced and secreted functional active chitinase, which degraded both natural and partially de-acetylated chitin. It had been suggested that the chitinase might be of importance for the host's environmental adaptability, which would influence its role as a pathogen [4] [5]. Investigation of chitin metabolism pathway would therefore help understand the wide ecological fitness of this pathogen. On the other hand, chitinase mediated degradation products of chitin and chitosan were also shown to be bioactive, which could have many potential applications in medicine and agriculture [3] [16]. As shown in this study, rChi-Ah would be a useful chitinolytic enzyme that may be applicable for both types of research.

Under conditions described in this study, rChi-Ah could be produced in large quantity; approximately 6 - 7 $\mathrm{mg}$ of rChi-Ah could be recovered from $100 \mathrm{ml}$ culture. Purified rChi-Ah was heat stable within the range of $37^{\circ} \mathrm{C}$ 
(a)

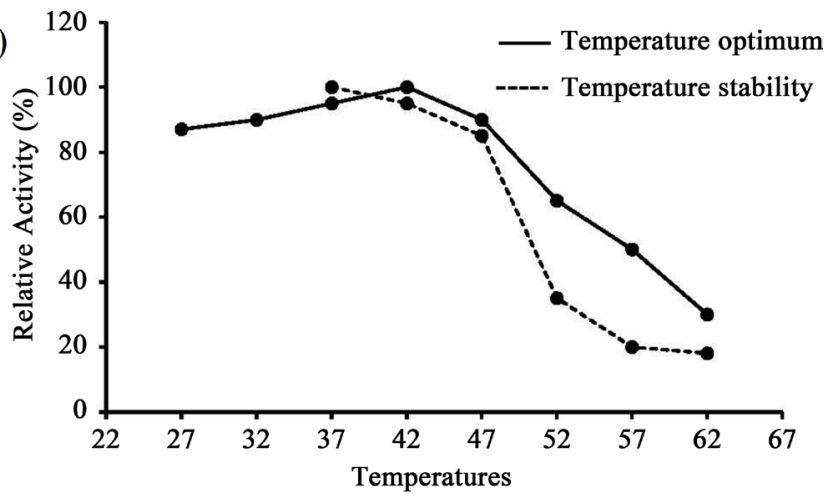

(b)

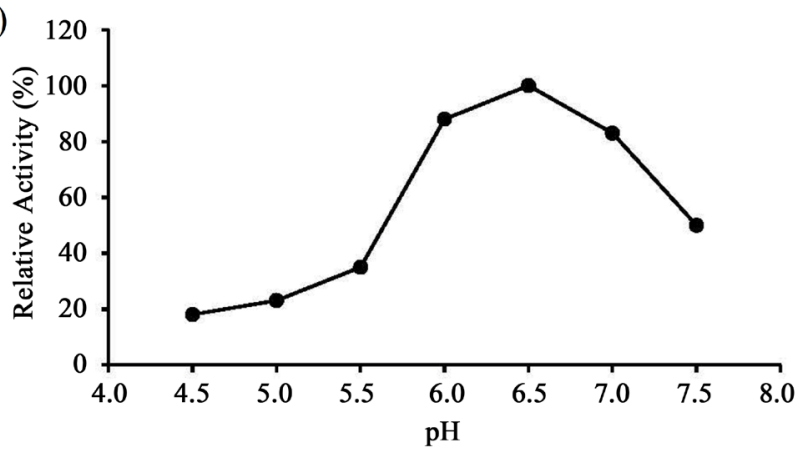

(c)

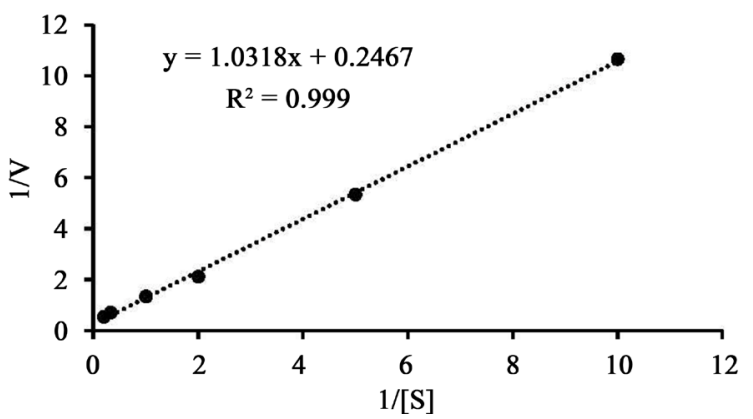

Figure 4. Thermostability, optima of temperature and $\mathrm{pH}$, and kinetics of rChi-Ah. (a) Heat stability and optimal temperature; (b) Optimal pH; (c) Calculation of Km and Vmax by Lineweaver-Burk plot (V-velocity and [S]—substrate concentration).

to $42^{\circ} \mathrm{C}$ and showed optimal activity at temperature of $42^{\circ} \mathrm{C}$ and $\mathrm{pH} 6.5$. These characteristics were similar to other bacterial chitinases studied [6] [17]. Enzymatically, rChi-Ah effectively hydrolyzed colloidal chitin to N,N'-diacetyl-glucosamine. The same product was observed in activity of a native chitinolytic enzyme purified from a pond-water isolate of Aeromonas sp [18] and a recombinant chitinase from Serratia marcescens [19]. Among many biological applications, N,N'-diacetyl-glucosamine was proved to be an inhibitor of lysozyme C (EC:3.2.1.17) and could be used to treat myocardial dysfunction in animals [20]. Related to this is the fact that lysozyme $\mathrm{C}$ is a defense enzyme of fish innate immune system and plays an important role in antibacterial infection [21]. Whether A. hydrophila has a metabolic pathway to produce N,N'-diacetyl-glucosamine to counteract fish’s lysozyme activity warrants further investigation.

For chitosan hydrolysis, the measured affinity of binding between rChi-Ah and chitosan (Km) and enzymatic reaction rate (Vmax) were similar to those of a native C4 bacterial chitinase [17]. LC-MS analysis revealed that rChi-Ah generated chitooligosaccharides with 2 - 4 units of glucosamine, all of which have at least one $\mathrm{N}$-acetyl group (Figure 3). N-acetylated glucosamine appears to be the recognition and cleavage site of rChi-Ah although no monomers (glucosamine or N-acetyl-glucosamine) were produced from both chitosan and chitin. The results are in agreement with the activity of a chitinase (ChiB) from Serratia marcescens [22] but different from that of 
ChiA of A. hydrophila strain SUWA, which produces both monomer and dimer from N-acetylchitooligomers [6]. The relative amounts of the five chitosan degradation products (Figure 3) are mostly dependent on the randomness and degree of de-acetylation of the chitosan chain. Since these chitooligosaccharides are found to have many interesting bioactivities [16], rChi-Ah will enrich the reservoir of chitinolytic enzymes to facilitate extensive research in related fields.

In conclusion, the chitinase of virulent $A$. hydrophila ML-10-51K was experimentally proved to be a functional active enzyme, which effectively degraded both chitin and chitosan. Identification of rChi-Ah mediated degradation products shed light on the possible role of A. hydrophila chitinase in pathogenicity and ecological fitness.

\section{Acknowledgements}

The authors thank Drs. Heping Cao and Perng-Kuang Chang for their editorial comments in the preparation of this manuscript and Ms. Ning Qin for her technical support. Mention of trade names or commercial products in this publication is solely for the purpose of providing specific information and does not imply recommendation or endorsement by the U.S. Department of Agriculture. This study was funded by USDA-ARS CRIS project \#6420-32000-024-00D.

\section{References}

[1] Rinaudo, M. (2006) Chitin and Chitosan: Properties and Applications. Progress in Polymer Science, 31, 603-632. http://dx.doi.org/10.1016/j.progpolymsci.2006.06.001

[2] Cohen-Kupiec, R. and Chet, I. (1998) The Molecular Biology of Chitin Digestion. Current Opinion in Biotechnology, 9, 270-277. http://dx.doi.org/10.1016/S0958-1669(98)80058-X

[3] Bhattacharya, D., Nagpure, A. and Gupta, R.K. (2007) Bacterial Chitinases: Properties and Potential. Critical Review in Biotechnology, 27, 21-28. http://dx.doi.org/10.1080/07388550601168223

[4] Pemberton, J.M., Kidd, S.P. and Schmidt, R. (1997) Secreted Enzymes of Aeromonas. FEMS Microbiology Letters, 152, 1-10. http://dx.doi.org/10.1111/j.1574-6968.1997.tb10401.x

[5] Roffey, P.E. and Pemberton, J.M. (1990) Cloning and Expression of an Aeromonas hydrophila Chitinase Gene in Escherichia Coli. Current Microbiology, 21, 329-337. http://dx.doi.org/10.1007/BF02092100

[6] Lan, X., Zhang, X., Hu, J. and Shimosaka, M. (2006) Cloning, Expression, and Characterization of a Chitinase from the Chitinolytic Bacterium Aeromonas hydrophila Strain SUWA-9. Bioscience Biotechnology, and Biochemistry, 70, 2437-2442. http://dx.doi.org/10.1271/bbb.60169

[7] Zhang, D., Pridgeon, J.W. and Klesius, P.H. (2014) Vaccination of Channel Catfish with Extracellular Products of Aeromonas hydrophila Provides Protection against Infection by the Pathogen. Fish and Shellfish Immunology, 36, 270-275. http://dx.doi.org/10.1016/j.fsi.2013.11.015

[8] Hossain, M.J., Waldbieser, G.C., Sun, D., Capps, N.K., Hemstreet, W.B., Carlisle, K., Griffin, M.J., Khoo, L., Goodwin, A.E, Sonstegard, T,S., Schroeder, S., Hayden, K., Newton, J.C., Terhune, J.S. and Liles, M.R. (2013) Implication of Lateral Genetic Transfer in the Emergence of Aeromonas hydrophila Isolates of Epidemic Outbreaks in Channel Catfish. PLoS ONE, 8, e80943. http://dx.doi.org/10.1371/journal.pone.0080943

[9] Pridgeon, J.W., Klesius, P.H., Song, L, Zhang, D., Kojima, K. and Mobley, J.A. (2013) Identification, Virulence, and mass Spectrometry of Toxic ECP Fractions of West Alabama Isolates of Aeromonas hydrophila Obtained from a 2010 Disease Outbreak. Veterinary Microbiology, 164, 336-343. http://dx.doi.org/10.1016/j.vetmic.2013.02.020

[10] Tekedar, H.C., Waldbieser, G.C., Kars, A., Liles, M.R., Griffin, M.J., Vamenta, S., Sonstegard, T., Hossain, M., Schroeder, S.G., Khoo, L. and Lawrence, M.L. (2013) Complete Genome Sequence of a Channel Catfish Epidemic Isolate, Aeromonas hydrophila Strain ML09-119. Genome Announcements, 1, e00755-13. http://dx.doi.org/10.1128/genomea.00755-13

[11] Murthy, N. and Bleakley, B. (2012) Simplified Method of Preparing Colloidal Chitin Used for Screening of ChitinaseProducing Microorganisms. Internet Scientific Publications, 10, 2.

[12] Seitzman, B. (2008) Colloidal Chitin Protocol. http://www.vyoma108.blogspot.com/2008/01/colloidal-chitin-protocol.html

[13] Bond, D.R., Tsai, B.M. and Russell, J.B. (1999) Physiological Characterization of Streptococcus bovis Mutants That Can Resist 2-Deoxyglucose-Induced Lysis. Microbiology, 145, 2977-2985.

[14] Jue, C.K. and Lipke, P.N. (1985) Determination of Reducing Sugars in the Nanomole Range with Tetrazolium Blue. Journal of Biochemical and Biophysical Methods, 11, 109-115. http://dx.doi.org/10.1016/0165-022X(85)90046-6 
[15] Seshadri, R., Joseph, S.W., Chopra, A.K., Sha, J., Shaw, J., Graf, J., Haft, D., Wu, M., Ren, Q., Rosovitz, M.J., Madupu, R., Tallon, L., Kim, M., Jin, S., Vuong, H., Stine, O.C., Ali, A., Horneman, A.J. and Heidelberg, J.F. (2006) Genome Sequence of Aeromonas hydrophila ATCC 7966T: Jack of All Trades. Journal of Bacteriology, 188, 8272-8282. http://dx.doi.org/10.1128/JB.00621-06

[16] Aam, B.B., Heggset, E.B., Norberg, A.L., Sørlie, M., Vårum, K.M. and Eijsink, V.G.H. (2010) Production of Chitooligosaccharides and Their Potential Applications in Medicine. Marine Drugs, 8, 1482-1517. http://dx.doi.org/10.3390/md8051482

[17] Tao, Y., Jin, H., Long, Z., Zhang, L., Ding, X., Tao, K., Ge, S. and Liu, S. (2005) Purification and Characterization of an Extracellular Chitinase Produced by Bacterial C4. Annals of Microbiology, 55, 213-218.

[18] Huang, J.H., Chen, C.J. and Su, Y.C. (1996) Production of Chitinolytic Enzymes from a Novel Species of Aeromonas. Journal of Industrial Microbiology, 17, 89-95. http://dx.doi.org/10.1007/BF01570049

[19] Wu, Y.J., Cheng, C.Y. and Li, Y.K. (2009) Cloning and Expression of Chitinase A from Serratia marcescens for Large-Scale Preparation of $N, N$-Diacetyl Chitobiose. Journal of Chinese Chemical Society-Taipei, 56, 688-695. http://dx.doi.org/10.1002/jccs.200900103

[20] Mink, S.N., Kasian, K., Jacobs, H., Cheng, Z.Q. and Light, R.B. (2008) N,N’-Diacetylchitibiose, an Inhibitor of Lysozyme, Reverses Myocardial Depression and Lessens Norepinephrine Requirements in Escherichia coli Sepsis in Dogs. Shock, 29, 681-687.

[21] Yu, L., Sun, B., Li, J. and Sun, L. (2013) Characterization of a c-Type Lysozyme of Scophthalmus maximus: Expression, Activity, and Antibacterial Effect. Fish and Shellfish Immunology, 34, 46-54. http://dx.doi.org/10.1016/j.fsi.2012.10.007

[22] Sørbotten, A., Horn, S.J., Eijsink, V.G.H. and Vårum, K.M. (2005) Degradation of Chitosans with Chitinase B from Serratia marcescens: Production of Chito-Oligosaccharides and Insight into Enzyme Processivity. The FEMS Journal, 272, 538-549. http://dx.doi.org/10.1111/j.1742-4658.2004.04495.x 